\title{
A VERSION OF ZHONG'S COERCIVITY RESULT FOR A GENERAL CLASS OF NONSMOOTH FUNCTIONALS
}

\author{
D. MOTREANU, V. V. MOTREANU, AND D. PAŞCA
}

Received 27 October 2001

A version of Zhong's coercivity result (1997) is established for nonsmooth functionals expressed as a sum $\Phi+\Psi$, where $\Phi$ is locally Lipschitz and $\Psi$ is convex, lower semicontinuous, and proper. This is obtained as a consequence of a general result describing the asymptotic behavior of the functions verifying the above structure hypothesis. Our approach relies on a version of Ekeland's variational principle. In proving our coercivity result we make use of a new general Palais-Smale condition. The relationship with other results is discussed.

\section{Introduction}

In this paper, we deal with the class of nonsmooth functionals $I: X \rightarrow \mathbb{R} \cup\{+\infty\}$ on a Banach space $X$ of the form

$$
I=\Phi+\Psi
$$

with $\Phi: X \rightarrow \mathbb{R}$ locally Lipschitz and $\Psi: X \rightarrow \mathbb{R} \cup\{+\infty\}$ convex, lower semicontinuous (1.s.c.), and proper (i.e., $\not \equiv+\infty)$.

For the functional $I$ as in (1.1), it was given in [9] the following definition of Palais-Smale (PS) condition.

Definition 1.1. The functional $I: X \rightarrow \mathbb{R} \cup\{+\infty\}$ in (1.1) satisfies the PS condition if every sequence $\left(u_{n}\right) \subset X$ with $I\left(u_{n}\right)$ bounded and for which there exists a sequence $\left(\epsilon_{n}\right) \subset \mathbb{R}^{+}, \epsilon_{n} \rightarrow 0^{+}$, such that

$$
\Phi^{0}\left(u_{n} ; v-u_{n}\right)+\Psi(v)-\Psi\left(u_{n}\right) \geq-\epsilon_{n}\left\|v-u_{n}\right\|, \quad \forall v \in X, \forall n,
$$

contains a (strongly) convergent subsequence in $X$. 
The notation $\Phi^{0}$ in (1.2) stands for the generalized directional derivative of the locally Lipschitz functional $\Phi: X \rightarrow \mathbb{R}$ introduced by Clarke [5] as follows:

$$
\Phi^{0}(u ; v)=\limsup _{\substack{w \rightarrow u \\ t \rightarrow 0^{+}}} \frac{1}{t}(\Phi(w+t v)-\Phi(w)), \quad \forall u, v \in X
$$

In the case where $\Phi \in C^{1}(X ; \mathbb{R})$ and $\Psi=0$, Definition 1.1 reduces to the usual PS condition. If $\Phi$ is locally Lipschitz and $\Psi=0$, Definition 1.1 expresses the PS condition in the sense of Chang [4]. If $\Phi \in C^{1}(X ; \mathbb{R})$ and $\Psi$ is convex, l.s.c., and proper, Definition 1.1 represents the concept of PS condition introduced by Szulkin [10].

A different extension of the usual PS condition is the following one.

Definition 1.2 (Zhong [11]). Let $h:[0,+\infty) \rightarrow[0,+\infty)$ be a continuous nondecreasing function such that $\int_{0}^{+\infty}(1 /(1+h(r))) d r=+\infty$. An l.s.c. proper function $\Phi: X \rightarrow \mathbb{R} \cup\{+\infty\}$ which is Gâteaux differentiable on its effective domain satisfies the PS condition if every sequence $\left(u_{n}\right) \subset X$ with $\Phi\left(u_{n}\right)$ bounded and

$$
\left\|\Phi^{\prime}\left(u_{n}\right)\right\|\left(1+h\left(\left\|u_{n}\right\|\right)\right) \longrightarrow 0 \quad \text { as } n \longrightarrow+\infty
$$

has a (strongly) convergent subsequence in $X$.

If $h=0$, Definition 1.2 reduces to the classical PS condition. In the case where $h(t)=t$, for all $t \geq 0$, Definition 1.2 coincides with the PS condition in the sense of Cerami [3].

It is natural to look for a concept of PS condition for functionals of type (1.1) incorporating simultaneously the two definitions above.

Definition 1.3. The functional $I: X \rightarrow \mathbb{R} \cup\{+\infty\}$ in (1.1) satisfies the PS condition if every sequence $\left(u_{n}\right) \subset X$ with $I\left(u_{n}\right)$ bounded and for which there exists a sequence $\left(\epsilon_{n}\right) \subset \mathbb{R}^{+}, \epsilon_{n} \rightarrow 0^{+}$, such that

$$
\Phi^{0}\left(u_{n} ; v-u_{n}\right)+\Psi(v)-\Psi\left(u_{n}\right) \geq-\frac{\epsilon_{n}}{1+h\left(\left\|u_{n}\right\|\right)}\left\|v-u_{n}\right\|, \quad \forall v \in X, \quad \forall n,
$$

contains a (strongly) convergent subsequence in $X$.

If $h=0$, Definition 1.3 reduces to Definition 1.1. If $\Phi \in C^{1}(X ; \mathbb{R})$ and $\Psi=0$, Definition 1.3 coincides with Definition 1.2 since relation (1.5) becomes (1.4) in this case. We point out that for $h(t)=t$, for all $t \geq 0$, Definition 1.3 expresses the extension of the PS condition in the sense of Cerami [3] to the class of nonsmooth functionals in (1.1).

A problem that has been extensively studied was the relationship between the PS condition and coercivity. We recall that a functional $I: X \rightarrow \mathbb{R} \cup\{+\infty\}$ is said to be coercive if the following property holds:

$$
I(u) \longrightarrow+\infty \quad \text { as }\|u\| \longrightarrow+\infty .
$$


The basic assertion in this direction is that, generally, the PS condition implies the coercivity. The first such result is the one of Caklovic et al. [2] who established this property for a functional $I: X \rightarrow \mathbb{R}$ which is l.s.c., Gâteaux differentiable, and satisfying the classical PS condition (see also Brézis and Nirenberg [1] for continuously differentiable functionals). The first result of this type, for nondifferentiable functionals, is due to Goeleven [7] who has shown the coercivity property in the case where the functional $I: X \rightarrow \mathbb{R} \cup\{+\infty\}$ has the structure (1.1) with $\Phi$ l.s.c. and Gâteaux differentiable, and $\Psi$ convex, l.s.c., and proper such that the PS condition in the sense of Szulkin [10] is satisfied. For nonsmooth functionals of the general form (1.1), an analogous result has been obtained in [8] making use of the PS condition stated in Definition 1.1. The corresponding property for nonsmooth functionals, satisfying the PS condition formulated in Definition 1.2, has been given by Zhong [11]. The aim of this paper is to prove the coercivity for the nonsmooth functionals verifying (1.1) together with the PS condition given in Definition 1.3.

In this paper, the coercivity assertion is obtained as a consequence of Theorem 2.3 below expressing the asymptotic behavior of a nonsmooth functional of type (1.1). Specifically, the coercivity property is derived from Theorem 2.3 by assuming the PS condition as formulated in Definition 1.3.

Theorem 2.3 cannot be deduced from Zhong's corresponding result [11, Theorem 3.7] because, generally, the functionals of type (1.1), which we consider, are not Gâteaux differentiable. However, Theorem 2.3 is not an extension of Theorem 3.7 in Zhong [11] because a Gâteaux differentiable, l.s.c., and proper functional is not necessarily of form (1.1). Our Theorem 2.3 represents the version of Zhong's corresponding result for a nonsmooth functional fulfilling the structure assumption (1.1).

The method of proof for Theorem 2.3 relies, as in the case of [11, Theorem 3.7], on Zhong's variational principle [11, Theorem 2.1] which is an extension of Ekeland's variational principle [6]. The proof of Theorem 2.3 takes into account, essentially, the structure of functionals in (1.1). To the end of rigourously proving Theorem 2.3, we slightly extend Zhong's variational principle [11, Theorem 2.1] in Theorem 2.1 below. The main idea is to allow the reference point $x_{0}$ to be in a larger space. Precisely, this extension is necessary because in Theorem 2.3 we encounter the situation where $x_{0}=0$ does not belong to the space $M_{0}$, on which the variational principle must be applied. Our argument corrects a small gap in the proof of [11, Theorem 3.7] concerning the mentioned difficulty. Furthermore, in comparison with Zhong's paper [11], our approach in Theorem 2.3 makes other improvements, among them, the accurate treatment of the passage from the $(N-1)$ th to the $N$ th step. Moreover, our hypotheses in Theorem 2.3 and Corollary 2.4 either are slightly weaker (see (2.8)) or give the correct requirement for making the proof (see (2.5)).

The rest of the paper is organized as follows. Section 2 is devoted to the statements of the results. Section 3 contains the proof of the main result. 
604 A version of Zhong's coercivity result

\section{Main results}

We start with a slight extension of Zhong's variational principle in [11].

Theorem 2.1. Let $h:[0,+\infty) \rightarrow[0,+\infty)$ be a continuous nondecreasing function such that

$$
\int_{0}^{+\infty} \frac{1}{1+h(r)} d r=+\infty
$$

Let $M$ be a closed subset of a complete metric space $\widetilde{M}$ endowed with the metric $d$, let a point $x_{0} \in \widetilde{M}$, and let $f: M \rightarrow \mathbb{R} \cup\{+\infty\}$ be an l.s.c., proper function which is bounded from below. Then, for all $\epsilon>0, v \in M$ with

$$
f(v)<\inf _{M} f+\epsilon
$$

and $\lambda>0$, there exists a point $w_{\epsilon, \lambda} \in M$ such that

$$
\begin{gathered}
f\left(w_{\epsilon, \lambda}\right) \leq f(v), \quad d\left(w_{\epsilon, \lambda}, x_{0}\right) \leq \bar{r}+r_{0}, \\
f(u) \geq f\left(w_{\epsilon, \lambda}\right)-\frac{\epsilon}{\lambda\left(1+h\left(d\left(x_{0}, w_{\epsilon, \lambda}\right)\right)\right)} d\left(u, w_{\epsilon, \lambda}\right), \quad \forall u \in M,
\end{gathered}
$$

where $r_{0}=d\left(x_{0}, v\right)$ and $\bar{r}$ verifies

$$
\int_{r_{0}}^{r_{0}+\bar{r}} \frac{1}{1+h(r)} d r \geq \lambda
$$

Proof. We endow $M$ with the metric induced by the one on $\widetilde{M}$, so $M$ becomes a complete metric space. A careful examination of the proof of [11, Theorem $2.1]$ shows that the argument therein can be carried out with any point $x_{0}$ in $\widetilde{M}$. Following the same lines as in the proof of [11, Theorem 2.1] (which goes back to Ekeland [6]), we achieve the stated conclusion.

Remark 2.2. The classical Ekeland's variational principle [6] is obtained from Theorem 2.1 taking $\widetilde{M}=M, h=0, x_{0}=v$, and $\bar{r}=\lambda$.

Our main result is the following theorem.

Theorem 2.3. Let $X$ be a Banach space and let a functional $I: X \rightarrow \mathbb{R} \cup\{+\infty\}$ be of type (1.1), that is, $I=\Phi+\Psi$ with $\Phi: X \rightarrow \mathbb{R}$ locally Lipschitz and $\Psi: X \rightarrow$ $\mathbb{R} \cup\{+\infty\}$ convex and l.s.c. If

$$
\alpha:=\liminf _{\|v\| \rightarrow+\infty} I(v) \in \mathbb{R}
$$

then for every sequence $\left(\epsilon_{n}\right) \subset \mathbb{R}^{+}$with $\epsilon_{n} \rightarrow 0^{+}$, there exists a sequence $\left(u_{n}\right) \subset X$ satisfying (1.5) (with a function $h:[0,+\infty) \rightarrow[0,+\infty)$ nondecreasing, continuous, 
and verifying (2.1)),

$$
\begin{array}{ll}
\left\|u_{n}\right\| \longrightarrow+\infty & \text { as } n \longrightarrow+\infty, \\
I\left(u_{n}\right) \longrightarrow \alpha & \text { as } n \longrightarrow+\infty .
\end{array}
$$

The proof will be done in Section 3.

Now we apply Theorem 2.3 for studying the coercivity of nonsmooth functionals in (1.1).

Corollary 2.4. Assume that the functional $I: X \rightarrow \mathbb{R} \cup\{+\infty\}$ satisfies the structure hypothesis (1.1) with $\Phi: X \rightarrow \mathbb{R}$ locally Lipschitz and $\Psi: X \rightarrow \mathbb{R} \cup\{+\infty\}$ convex, l.s.c., and proper. If I verifies the PS condition in Definition 1.3 and

$$
\liminf _{\|v\| \rightarrow+\infty} I(v)>-\infty
$$

then I is coercive, that is property (1.6) holds.

Proof. Suppose, by contradiction, that $I$ is not coercive. In view of (2.8), this is equivalent to (2.5) which enables us to apply Theorem 2.3. Corresponding to a sequence $\epsilon_{n} \rightarrow 0^{+}$, we find a sequence $\left(u_{n}\right) \subset X$ fulfilling (1.5), (2.6), and (2.7). On the basis of (1.5), (2.7), and PS condition in the sense of Definition 1.3, it follows that there exists a subsequence of $\left(u_{n}\right)$ which is strongly convergent in $X$. Thus, we arrived at a contradiction with (2.6). The proof is complete.

\section{Proof of Theorem 2.3}

Note that the condition $\alpha \in \mathbb{R}$, imposed in (2.5), implies that the functional $I$ is proper outside every ball in $X$. We fix a positive number $\epsilon \leq 1 / 3$. Define the function

$$
m(r)=\inf _{\|u\| \geq r} I(u), \quad r>0 .
$$

The function $m$ in (3.1) is nondecreasing and it satisfies

$$
\lim _{r \rightarrow+\infty} m(r)=\alpha .
$$

From (3.2), there exists a number $\bar{r}=\bar{r}(\epsilon) \geq 1 / \epsilon$ such that

$$
\alpha-\epsilon^{2} \leq m(r), \quad \forall r \geq \bar{r} .
$$

Using property (2.1) of the function $h$, it follows that

$$
\int_{\bar{r}}^{+\infty} \frac{1}{1+h(r)} d r=+\infty .
$$

Therefore, we can choose $r^{*}>\bar{r}$ such that

$$
\int_{\bar{r}}^{r^{*}} \frac{1}{1+h(r)} d r \geq 1 .
$$


Corresponding to $\epsilon>0$, the definition of the integral yields a partition $\bar{r}=r_{N}<$ $r_{N-1}<\cdots<r_{1}<r_{0}=r^{*}$ for which one has

$$
\left|\int_{\bar{r}}^{r^{*}} \frac{1}{1+h(r)} d r-\sum_{k=0}^{N-1} \frac{1}{1+h\left(r_{k}\right)}\left(r_{k}-r_{k+1}\right)\right|<\epsilon .
$$

We consider the following sets:

$$
M_{k}=\left\{u \in X:\|u\| \geq r_{k}\right\}, \quad 0 \leq k \leq N .
$$

The requirements in Theorem 2.1 are fulfilled with $M=M_{0}, \widetilde{M}=X, f=I$, and $x_{0}=0$. To justify this, we notice that $M_{0}$ is a closed subset of the Banach space $X$. The functional $I: X \rightarrow \mathbb{R} \cup\{+\infty\}$, expressed in (1.1), is l.s.c. and proper on $M_{0}$. Finally, by (3.1) and (3.3) we derive that

$$
I(u) \geq m(\|u\|) \geq \alpha-\epsilon^{2}, \quad \forall u \in X,\|u\| \geq \bar{r},
$$

which ensures that the functional $I$ is bounded from below on $M_{0}$ since $\bar{r}<r_{0}$. Then, Theorem 2.1 (with $\epsilon$ replaced by $\epsilon^{2}$ and $\lambda=\epsilon$ ) provides a point $w_{0}=$ $w_{0}(\epsilon) \in M_{0}$ such that

$$
I\left(w_{0}\right)<m\left(r_{0}\right)+\epsilon^{2} \leq \alpha+\epsilon^{2}
$$

(see also (3.2)) and

$$
I(u) \geq I\left(w_{0}\right)-\frac{\epsilon}{1+h\left(\left\|w_{0}\right\|\right)}\left\|u-w_{0}\right\|, \quad \forall u \in M_{0} .
$$

Assume that

$$
\left\|w_{0}\right\|>r_{0} \quad \text { or } \quad \text { (3.10) holds for every } u \in M_{1} \text {. }
$$

For an arbitrary $w \in X$, it is permitted to set in (3.10) $u=w_{0}+t\left(w-w_{0}\right)$ with $t>0$ sufficiently small. Then, we can write

$$
\begin{aligned}
\Phi\left(w_{0}+t\left(w-w_{0}\right)\right)+\Psi\left(w_{0}+t\left(w-w_{0}\right)\right) \\
\geq \Phi\left(w_{0}\right)+\Psi\left(w_{0}\right)-\frac{\epsilon t}{1+h\left(\left\|w_{0}\right\|\right)}\left\|w-w_{0}\right\| .
\end{aligned}
$$

On the basis of convexity of $\Psi: X \rightarrow \mathbb{R} \cup\{+\infty\}$, the inequality above yields

$$
\begin{gathered}
\Phi\left(w_{0}+t\left(w-w_{0}\right)\right)-\Phi\left(w_{0}\right)+t\left(\Psi(w)-\Psi\left(w_{0}\right)\right) \\
\geq-\frac{\epsilon t}{1+h\left(\left\|w_{0}\right\|\right)}\left\|w-w_{0}\right\| .
\end{gathered}
$$


Dividing by $t>0$ and then passing to the upper limit as $t \rightarrow 0^{+}$, we obtain that

$$
\begin{gathered}
\limsup _{t \rightarrow 0^{+}} \frac{1}{t}\left(\Phi\left(w_{0}+t\left(w-w_{0}\right)\right)-\Phi\left(w_{0}\right)\right)+\Psi(w)-\Psi\left(w_{0}\right) \\
\geq-\frac{\epsilon}{1+h\left(\left\|w_{0}\right\|\right)}\left\|w-w_{0}\right\| .
\end{gathered}
$$

We deduce that

$$
\Phi^{0}\left(w_{0} ; w-w_{0}\right)+\Psi(w)-\Psi\left(w_{0}\right) \geq-\frac{\epsilon}{1+h\left(\left\|w_{0}\right\|\right)}\left\|w-w_{0}\right\|, \quad \forall w \in X
$$

Since $w_{0} \in M_{0}$, it is known that $\left\|w_{0}\right\| \geq r_{0}=r^{*} \geq 1 / \epsilon$. From (3.8) and (3.9) we infer that $\alpha-\epsilon^{2} \leq I\left(w_{0}\right)<\alpha+\epsilon^{2}$. Taking into account that the assertions above are valid for an arbitrary $\epsilon \in(0,1 / 3)$, we see that properties (1.5), (2.6), and (2.7) are proved under the additional assumption (3.11).

We may, thus, suppose that

$$
\left\|w_{0}\right\|=r_{0}
$$

and there exists some $\overline{u_{1}} \in M_{1} \backslash M_{0}$ such that

$$
I\left(\overline{u_{1}}\right)<I\left(w_{0}\right)-\frac{\epsilon}{1+h\left(\left\|w_{0}\right\|\right)}\left\|\overline{u_{1}}-w_{0}\right\| .
$$

Using the construction leading to [11, relation (2.7)] with $M_{1}, \epsilon^{2}$ and $\epsilon$ in place of $M, \epsilon$ and $\lambda$, respectively, and choosing $u_{1}^{1}=w_{0}$, there exists a sequence $\left(u_{n}^{1}\right) \subset$ $M_{1}$ such that

$$
I\left(u_{n+1}^{1}\right) \leq I\left(u_{n}^{1}\right)-\frac{\epsilon}{1+h\left(\left\|u_{n}^{1}\right\|\right)}\left\|u_{n}^{1}-u_{n+1}^{1}\right\|, \quad \forall n \geq 1 .
$$

Arguing as in the proof of [11, Theorem 2.1], on the basis of relation (3.18), we deduce the existence of a point $w_{1} \in M_{1}$ with the properties

$$
\begin{gathered}
\lim _{n \rightarrow+\infty} u_{n}^{1}=w_{1}, \\
I(u) \geq I\left(w_{1}\right)-\frac{\epsilon}{1+h\left(\left\|w_{1}\right\|\right)}\left\|u-w_{1}\right\|, \quad \forall u \in M_{1} .
\end{gathered}
$$

In addition, by (3.19) and the lower semicontinuity of $I$ in conjunction with (3.18) and (3.9), we obtain that

$$
I\left(w_{1}\right) \leq \liminf _{n \rightarrow+\infty} I\left(u_{n}^{1}\right)=\lim _{n \rightarrow+\infty} I\left(u_{n}^{1}\right) \leq I\left(u_{1}^{1}\right)<\alpha+\epsilon^{2} .
$$


Relations (3.21) and (3.20) show that we arrived at a situation which is similar to the one described in the previous step, that is, (3.9) and (3.10). In this respect, if

$$
\left\|w_{1}\right\|>r_{1} \quad \text { or } \quad(3.20) \text { holds for every } u \in M_{2}
$$

(an assertion analogous to (3.11)), we complete the proof as above.

It remains to consider the case where (3.22) does not hold, that is,

$$
\left\|w_{1}\right\|=r_{1}
$$

and there exists some $\overline{u_{2}} \in M_{2} \backslash M_{1}$ such that

$$
I\left(\overline{u_{2}}\right)<I\left(w_{1}\right)-\frac{\epsilon}{1+h\left(\left\|w_{1}\right\|\right)}\left\|\overline{u_{2}}-w_{1}\right\| .
$$

Thanks to relations (3.23) and (3.24), we are in the same situation as (3.16) and (3.17), hence, we may pass to the next step.

Continuing the process, by the construction around [11, relation (2.7)], we find a convergent sequence $\left(u_{n}^{k}\right) \subset M_{k}, 1 \leq k \leq N$, with $u_{1}^{k}=w_{k-1}$, satisfying

$$
I\left(u_{n+1}^{k}\right) \leq I\left(u_{n}^{k}\right)-\frac{\epsilon}{1+h\left(\left\|u_{n}^{k}\right\|\right)}\left\|u_{n}^{k}-u_{n+1}^{k}\right\|, \quad \forall n \geq 1
$$

Moreover, there exists

$$
\lim _{n \rightarrow+\infty} u_{n}^{k}=w_{k}
$$

with the property

$$
I(u) \geq I\left(w_{k}\right)-\frac{\epsilon}{1+h\left(\left\|w_{k}\right\|\right)}\left\|u-w_{k}\right\|, \quad \forall u \in M_{k} .
$$

Now, two situations could arise: either

$$
\left\|w_{k}\right\|>r_{k} \quad \text { or } \quad \text { (3.27) holds for every } u \in M_{k+1}
$$

(where we set $M_{N+1}=X$ ) or

$$
\left\|w_{k}\right\|=r_{k}
$$

and there is $\overline{u_{k+1}} \in M_{k+1} \backslash M_{k}$ with

$$
I\left(\overline{u_{k+1}}\right)<I\left(w_{k}\right)-\frac{\epsilon}{1+h\left(\left\|w_{k}\right\|\right)}\left\|\overline{u_{k+1}}-w_{k}\right\| .
$$

Now we prove that for at least one $0 \leq k \leq N$ we are in the situation described in (3.28). Clearly, this will accomplish the proof by means of a reasoning similar to the one below relation (3.11). 
We argue by contradiction. Suppose that

$w_{k}$ satisfies (3.27), (3.29), and (3.30), for every $0 \leq k \leq N$.

The contradiction will be achieved through the inequality

$$
\frac{\epsilon}{1+h\left(\left\|w_{k}\right\|\right)}\left\|w_{k}-w_{k+1}\right\| \leq I\left(w_{k}\right)-I\left(w_{k+1}\right), \quad \forall k, 0 \leq k \leq N-1 .
$$

Assume for a moment that (3.32) is valid. According to (3.32), (3.9), $w_{N} \in$ $M_{N}$, and (3.3), we derive that

$$
\sum_{k=0}^{N-1} \frac{\epsilon}{1+h\left(\left\|w_{k}\right\|\right)}\left\|w_{k}-w_{k+1}\right\| \leq I\left(w_{0}\right)-I\left(w_{N}\right)<\alpha+\epsilon^{2}-m\left(r_{N}\right) \leq 2 \epsilon^{2}
$$

Using (3.5), (3.6), (3.29), (3.31), and (3.33), it turns out that

$$
\begin{aligned}
1-\epsilon & \leq \int_{\bar{r}}^{r^{*}} \frac{1}{1+h(r)} d r-\epsilon<\sum_{k=0}^{N-1} \frac{1}{1+h\left(r_{k}\right)}\left(r_{k}-r_{k+1}\right) \\
& =\sum_{k=0}^{N-1} \frac{1}{1+h\left(\left\|w_{k}\right\|\right)}\left(\left\|w_{k}\right\|-\left\|w_{k+1}\right\|\right) \\
& \leq \sum_{k=0}^{N-1} \frac{1}{1+h\left(\left\|w_{k}\right\|\right)}\left\|w_{k}-w_{k+1}\right\| \leq 2 \epsilon .
\end{aligned}
$$

Thus, we get that $\epsilon>1 / 3$. This contradicts the choice $\epsilon \leq 1 / 3$ and completes the proof provided that (3.32) is true.

In order to check (3.32), we fix $k$ with $0 \leq k \leq N-1$. Note that if

$$
\left\|u_{j}^{k+1}\right\| \leq\left\|w_{k}\right\|=r_{k}, \quad \forall j \geq 1
$$

the claim in (3.32) is proved. Indeed, by (3.26), the lower semicontinuity of $I$ and (3.25), we may write

$$
\begin{aligned}
I\left(w_{k+1}\right) & \leq \liminf _{m \rightarrow+\infty} I\left(u_{m}^{k+1}\right) \leq I\left(u_{n}^{k+1}\right) \\
& \leq I\left(u_{1}^{k+1}\right)-\sum_{j=1}^{n-1} \frac{\epsilon}{1+h\left(\left\|u_{j}^{k+1}\right\|\right)}\left\|u_{j}^{k+1}-u_{j+1}^{k+1}\right\|, \quad \forall n \geq 2 .
\end{aligned}
$$


In addition, by means of equality $u_{1}^{k+1}=w_{k},(3.26)$, and the triangle inequality, we have

$$
\begin{aligned}
\left\|w_{k}-w_{k+1}\right\| & =\lim _{n \rightarrow+\infty}\left\|u_{1}^{k+1}-u_{n}^{k+1}\right\| \\
& \leq \lim _{n \rightarrow+\infty} \sum_{j=1}^{n-1}\left\|u_{j}^{k+1}-u_{j+1}^{k+1}\right\| \\
& =\sum_{j=1}^{+\infty}\left\|u_{j}^{k+1}-u_{j+1}^{k+1}\right\| .
\end{aligned}
$$

Making use of monotonicity of $h$ in conjunction with (3.35) and (3.36), the inequality above leads to

$$
\begin{aligned}
\frac{\epsilon}{1+h\left(\left\|w_{k}\right\|\right)}\left\|w_{k}-w_{k+1}\right\| & \leq \sum_{j=1}^{+\infty} \frac{\epsilon}{1+h\left(\left\|w_{k}\right\|\right)}\left\|u_{j}^{k+1}-u_{j+1}^{k+1}\right\| \\
& \leq \sum_{j=1}^{+\infty} \frac{\epsilon}{1+h\left(\left\|u_{j}^{k+1}\right\|\right)}\left\|u_{j}^{k+1}-u_{j+1}^{k+1}\right\| \\
& \leq I\left(w_{k}\right)-I\left(w_{k+1}\right) .
\end{aligned}
$$

This means that if (3.35) holds, inequality (3.32) is checked.

Property (3.35) is true for $j=1$ being verified with equality. Thus, for completing the proof it is sufficient to verify

$$
\left\|u_{j}^{k+1}\right\|<\left\|w_{k}\right\|=r_{k}, \quad \forall j \geq 2
$$

The proof of (3.39) is done by recurrence. Considering first the case $j=2$, we point out that the set

$$
\left\{u \in M_{k+1}: I(u)<I\left(w_{k}\right)-\frac{\epsilon}{1+h\left(\left\|w_{k}\right\|\right)}\left\|u-w_{k}\right\|\right\}
$$

is nonempty because it contains at least $\overline{u_{k+1}}$ (see (3.30) and (3.31)). Taking into account the equality $w_{k}=u_{1}^{k+1}$, by the construction of the sequence $\left(u_{n}^{k+1}\right)$, it is known that $u_{2}^{k+1}$ belongs to the set described by (3.40). Comparing (3.40) and (3.27), it follows that $u_{2}^{k+1} \notin M_{k}$ which reads as $\left\|u_{2}^{k+1}\right\|<r_{k}$. Assertion (3.39) is, thus, checked for $j=2$.

Assume inductively that for some $j>2$, we have $\left\|u_{n}^{k+1}\right\|<r_{k}$ with $2 \leq n \leq j-1$. We must verify that $\left\|u_{j}^{k+1}\right\|<r_{k}$. Arguing by contradiction, suppose $\left\|u_{j}^{k+1}\right\| \geq r_{k}$. This ensures that $u_{j}^{k+1} \neq u_{j-1}^{k+1}$, so by (3.25) with $n=j-1$ and (3.31), we infer 
that

$$
\begin{aligned}
I\left(u_{j}^{k+1}\right) & <I\left(u_{j-1}^{k+1}\right)-\frac{\epsilon}{1+h\left(\left\|u_{j-1}^{k+1}\right\|\right)}\left\|u_{j-1}^{k+1}-u_{j}^{k+1}\right\| \\
& \leq I\left(u_{1}^{k+1}\right)-\sum_{n=1}^{j-1} \frac{\epsilon}{1+h\left(\left\|u_{n}^{k+1}\right\|\right)}\left\|u_{n}^{k+1}-u_{n+1}^{k+1}\right\| .
\end{aligned}
$$

Since we admitted $\left\|u_{j}^{k+1}\right\| \geq r_{k}$ and using (3.27), we may write

$$
I\left(u_{j}^{k+1}\right) \geq I\left(w_{k}\right)-\frac{\epsilon}{1+h\left(\left\|w_{k}\right\|\right)}\left\|u_{j}^{k+1}-w_{k}\right\| .
$$

Relations (3.41), $u_{1}^{k+1}=w_{k}$, (3.42), the triangle inequality, $\left\|u_{n}^{k+1}\right\|<r_{k}=\left\|w_{k}\right\|$ for $2 \leq n \leq j-1$, and the increasing monotonicity of $h$ yield

$$
\begin{aligned}
\sum_{n=1}^{j-1} \frac{\epsilon}{1+h\left(\left\|u_{n}^{k+1}\right\|\right)}\left\|u_{n}^{k+1}-u_{n+1}^{k+1}\right\| & <I\left(u_{1}^{k+1}\right)-I\left(u_{j}^{k+1}\right) \\
& \leq \frac{\epsilon}{1+h\left(\left\|u_{1}^{k+1}\right\|\right)}\left\|u_{j}^{k+1}-u_{1}^{k+1}\right\| \\
& \leq \frac{\epsilon}{1+h\left(\left\|u_{1}^{k+1}\right\|\right)} \sum_{n=1}^{j-1}\left\|u_{n}^{k+1}-u_{n+1}^{k+1}\right\| \\
& \leq \sum_{n=1}^{j-1} \frac{\epsilon}{1+h\left(\left\|u_{n}^{k+1}\right\|\right)}\left\|u_{n}^{k+1}-u_{n+1}^{k+1}\right\| .
\end{aligned}
$$

The achieved contradiction implies that $\left\|u_{j}^{k+1}\right\|<r_{k}$. The inductive process is accomplished, thus (3.39) holds true. The proof is complete.

\section{References}

[1] H. Brézis and L. Nirenberg, Remarks on finding critical points, Comm. Pure Appl. Math. 44 (1991), no. 8-9, 939-963.

[2] L. Čaklović, S. J. Li, and M. Willem, A note on Palais-Smale condition and coercivity, Differential Integral Equations 3 (1990), no. 4, 799-800.

[3] G. Cerami, An existence criterion for the critical points on unbounded manifolds, Istit. Lombardo Accad. Sci. Lett. Rend. A 112 (1978), no. 2, 332-336.

[4] K.-C. Chang, Variational methods for nondifferentiable functionals and their applications to partial differential equations, J. Math. Anal. Appl. 80 (1981), no. 1, 102129.

[5] F. H. Clarke, Optimization and Nonsmooth Analysis, Canadian Mathematical Society Series of Monographs and Advanced Texts, John Wiley \& Sons, New York, 1983.

[6] I. Ekeland, On the variational principle, J. Math. Anal. Appl. 47 (1974), 324-353.

[7] D. Goeleven, A note on Palais-Smale condition in the sense of Szulkin, Differential Integral Equations 6 (1993), no. 5, 1041-1043.

[8] D. Motreanu and V. V. Motreanu, Coerciveness property for a class of non-smooth functionals, Z. Anal. Anwendungen 19 (2000), no. 4, 1087-1093. 


\section{A version of Zhong's coercivity result}

[9] D. Motreanu and P. D. Panagiotopoulos, Minimax Theorems and Qualitative Properties of the Solutions of Hemivariational Inequalities, Nonconvex Optimization and Its Applications, vol. 29, Kluwer Academic Publishers, Dordrecht, 1999.

[10] A. Szulkin, Minimax principles for lower semicontinuous functions and applications to nonlinear boundary value problems, Ann. Inst. H. Poincaré Anal. Non Linéaire 3 (1986), no. 2, 77-109.

[11] C.-K. Zhong, A generalization of Ekeland's variational principle and application to the study of the relation between the weak P.S. condition and coercivity, Nonlinear Anal. 29 (1997), no. 12, 1421-1431.

D. Motreanu: Département de Mathématiques, Université de Perpignan, 66860 Perpignan, France

E-mail address: motreanu@univ-perp.fr

V. V. Motreanu: Département de Mathématiques, Université de Perpignan, 66860 Perpignan, France

E-mail address: viorica@univ-perp.fr

D. Paşca: Mathematical Sciences Department, Worcester Polytechnic InstiTUTE, WORCESTER, MA 01609-2280, USA

E-mail address: dpasca@wpi.edu 


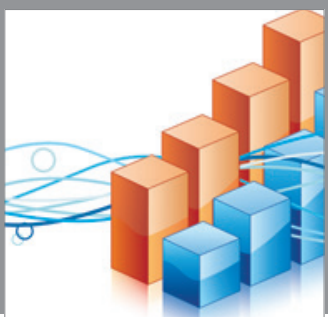

Advances in

Operations Research

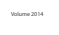

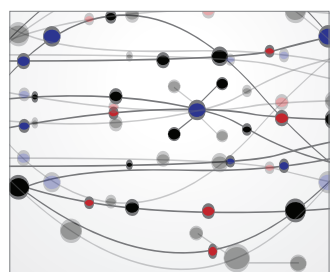

\section{The Scientific} World Journal
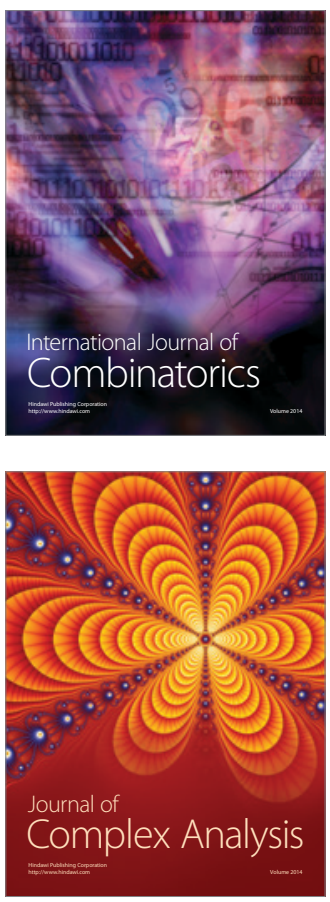

International Journal of

Mathematics and

Mathematical

Sciences
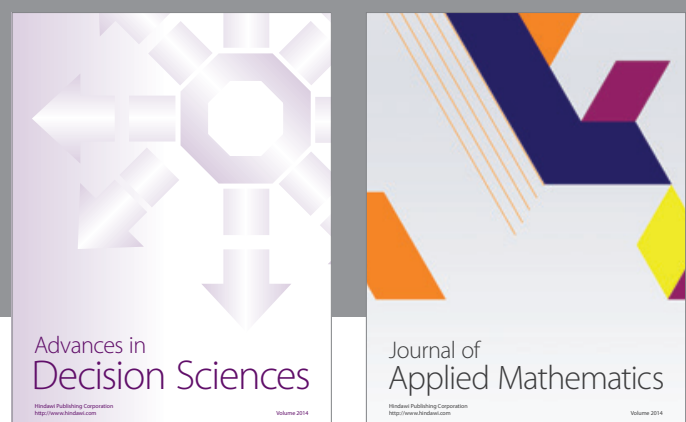

Journal of

Applied Mathematics
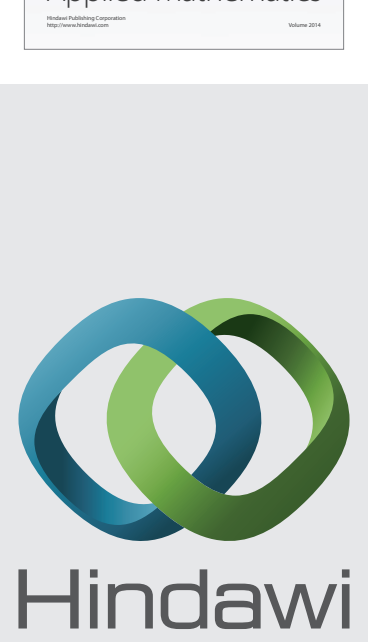

Submit your manuscripts at http://www.hindawi.com
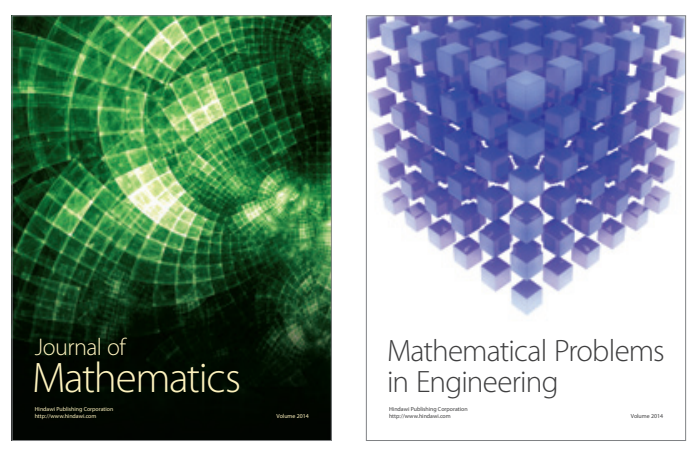

Mathematical Problems in Engineering
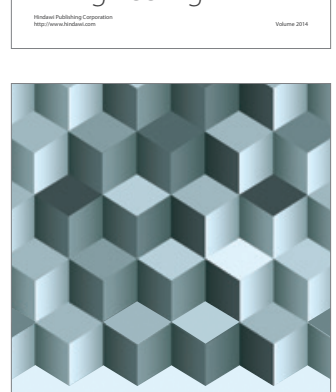

Journal of

Function Spaces
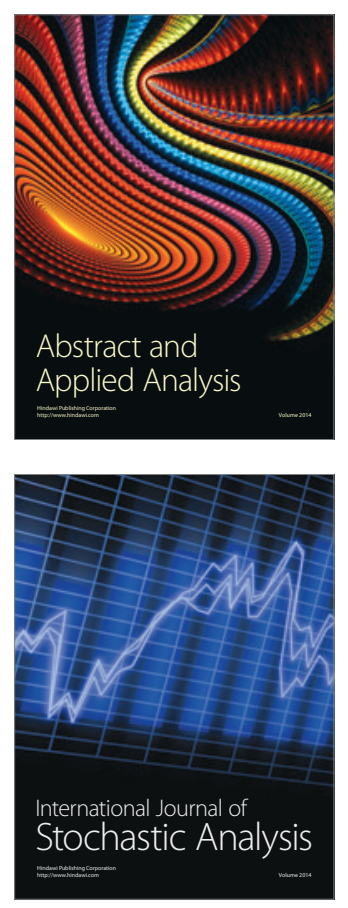

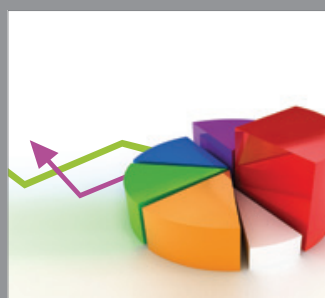

ournal of

Probability and Statistics

Promensencen
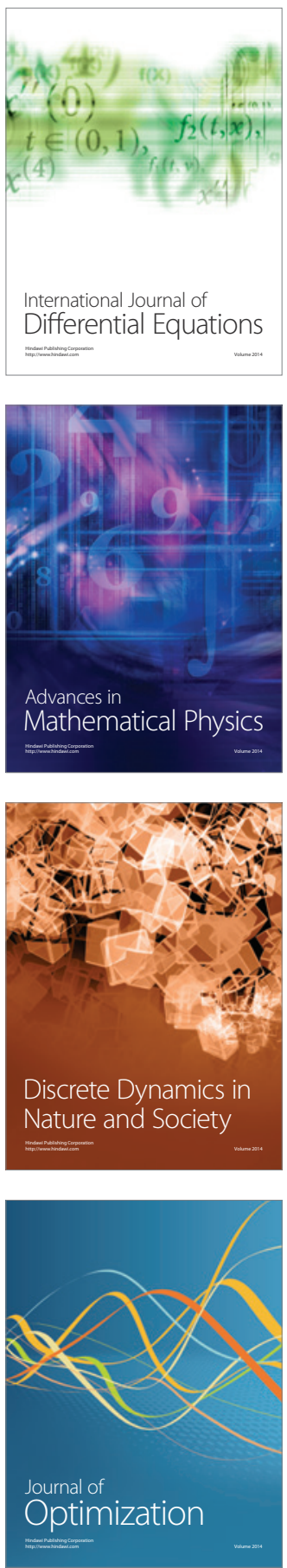